\title{
A project to get the Shroud image by scanning
}

\author{
Guido Belforte ${ }^{1, \mathrm{a}}$, Carlo Ferraresi ${ }^{1, \mathrm{~b}}$, Nello Balossino ${ }^{2, \mathrm{c}}$, and Sergio Rabellino ${ }^{2, \mathrm{~d}}$ \\ ${ }^{1}$ Dipartimento di Meccanica, Politecnico di Torino, Italy \\ ${ }^{2}$ Dipartimento di Informatica, Università di Torino, Italy
}

\begin{abstract}
The paper deals with a system able to perform a single uniform scan acquisition of the Shroud.
\end{abstract}

\section{Introduction}

Digital photographic images acquisition often feels pain of geometrical, spectral, and radiometric limits. This happens especially when objects of large size and with particular texture characteristics as well colorimetric values are managed [1].

As it is known, the Shroud is a linen cloth, approximately rectangular in shape, and with huge dimension: $441 \mathrm{~cm}$ in length and $113 \mathrm{~cm}$ in height. We want to point up that the Shroud is one of the few example of objects with a large extension offered by iconographic and pictorial fields.

The basic color of the Shroud cloth looks like ivory; the body print is perceived brown, as well as the burns, while the blood stains appear vermilion in color. Color differences cover a very narrow range and a lot of difficulties arise in reproducing them from physiological and physical point of view.

In addition to the characteristics mentioned above, it might be considered that the eidetic data are imprinted on a surface whose pattern looks like an herringbone: repetitions of holes and protrusions are present. This means that an approximately uniform ambient light used to acquire the image does not satisfy the request necessary to obtain suitable results from a radiometric and photographic point of view.

Moreover the optical instruments make distortions when used to acquire a big object with a single shot or by a set of tassels inter-connected.

\footnotetext{
a e-mail: Guido.Belforte@polito.it

b e-mail: Carlo.Ferraresi@polito.it

c e-mail: Nello.Balossino@unito.it

d e-mail: Sergio.Rabellino@unito.it
}

This is an Open Access article distributed under the terms of the Creative Commons Attribution License 4.0, which permits unrestricted use, distribution, and reproduction in any medium, provided the original work is properly cited. 


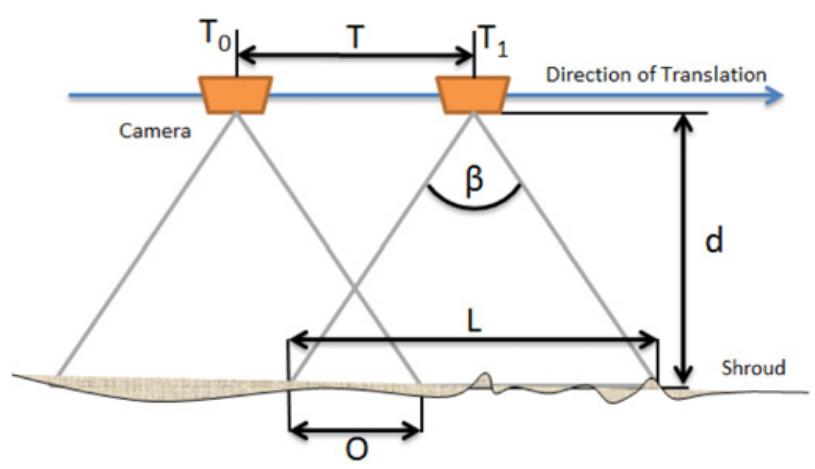

Figure 1. Geometry for image acquisition by camera translation.

\section{The Shroud images}

The photographic acquisitions of the Shroud realized until now $[2,3]$ have been based on one of the protocols mentioned below:

a) one analogical single shot: geometric errors arise from optical distortions, as well as from radiometric inaccuracy due to irregular lighting and film response; optical aberrations also introduce different levels of detail, decreasing from the center of the optics, to the areas of the border. The final results highlight the lack of color compatibility as well as the narrowness of details of the morphometric aspects on the impressed body, especially when magnification is used;

b) a narrow number of analogical photographic shots with digital conversion and subsequent handmade stitching: errors persist, because the correctness of the juxtaposition at the contact edges of the shots performed are not ensured. For each shot, the stitching operation produces a contact band between the shot and all the other neighbors shots; these bands are calculated mixing the image borders and there is no certainty that resulting pixels correspond to the real data. Moreover the chromatics is strongly penalized due to the different color characteristics of the shots; it is necessary to apply suitable methods in order to balance differences emerged in the acquisition, bringing uncertainty on the correspondence with the real data again;

c) a high number of shots obtained by high resolution scanner in A4 format: by now no single image has been obtained using these data;

d) variable amount of digital images merged for obtaining a global image by using stitching methods: questions arise about the correctness and validity of the juxtaposition in the morphometric image representation. The lighting model may also introduces colors not so close to real data.

\section{Stitching}

A simplified explanation of the problems arising when a set of tassels is managed by using stitching algorithms to obtain a total image is presented the following $[4,5]$.

For the Shroud image obtained applying the "stitch \& merge" method a camera translation movement is used: the camera is shifted in a parallel plane to the object one.

The total image is constructed by composing the image acquired with each translation of the camera until the series of images cover the entire object. Figure 1 shows the set-up of this method, where the camera is aligned with the sliding plate so that the imaging plane is parallel to the orientation of the sliding plate. 
For the purposes of this investigation, in order to simplify the debate, we can consider a movement onto one of the two main Cartesian axis.

Let $T$ the camera translation, $d$ the distance between camera and object, and $\beta$ the camera's horizontal angle of view, the ratio of the overlapping region of the composed image can be estimated by Eq. (1).

$$
\frac{O}{L}=1-\frac{T}{2 d \tan (\beta / 2)} .
$$

The equation shows that if we want every image acquired has the same sized overlapping region the translation $T$ and $d$ should vary proportionally.

In an ideal case the two adjacent images (tassels) must have the same intensity values for every pair of the same pixel in the overlapping region. In real cases, however, due to complex variation of the light illumination of the Shroud, or due to the camera point of view or even the angle between the camera and the light source, the intensities at the overlapping border might be different.

In addition, the intrinsic characteristics of the Shroud cloth like the herringbone texture, the folds, the holes, the patches, produce a profile with valley and mountains that could be viewed in a dissimilar way if acquired from different directions.

Starting from these practical evidences, many authors proposed algorithms in order to avoid the creation of seams along the contact area between two tassels in generic images. A seam is the artificial edge produced by the intensity differences of pixels immediately next to where the images are joined.

The purpose of these algorithms are generally two:

- the seams between images should be visually undetectable;

- the quality of the original tassels should be preserved as much as possible; the contact border between two tassels in the resultant image will be a sort of mixing of each corresponding pixel in the two tassels and it's adjacent.

The obtained image portrays a compromise between the original information taken from the Shroud and the visual pleasure of the image: the seams are mostly absent. This compromise could be acceptable if the purpose of the acquisition is the communication of the evocative nature of the Shroud with giant prints or any other media, but can be a severe constraint if we need to get the real and effective information from the cloth for scientific purposes.

To overcome the constraints described above, the proposed solution consists in a sort of asymptotic tassels acquisition method, were the $T$ and $\beta$ parameters are very small, until they cover a single pixel: we will have a tassels for every pixel. In real case the asymptotic method would lead to a single bar digital scanner.

The Shroud acquisition could be done with a single uniform scan operation along one direction of the cloth, at a reduced distance $d$, where the illumination model can be better controlled. It also useful to acquire the image with a wide spectrum, in order to catch particularly data object we could are interested on.

Taking into account the sensors technologies available today, the choose falls between two types:

- Contact Image Sensors (CIS);

- Reduction Type Linear Sensors, generally called CCD, acronym of Charge Coupled Device.

A Linear CIS sensor consists of an array of detectors, covered by focusing lenses and paired by red, green, and blue LEDs for illumination. In turn, every colored LED light is fired, and then reflected by the object and captured by a glass rod lens, and then directed towards the image sensors that capture the pixel.

The CIS sensor spans the entire scan line and has a 1:1 mapping between a pixel under the current scan line and the pixel in the image sensors. This make possible a very high geometrical accuracy; on 
the contrary the depth of focus is very small, usually a fraction of a millimeter. This is not suitable for scanning objects non completely flat like the cloth of the Shroud.

Moreover, the illumination generated by chromatic LEDs heavily limit the chromatic gamut of the acquired image: this is not acceptable when the object captured has plenty of shadows like the Shroud.

A Linear CCDs are single or multi line arrays of multispectral sensors able to capture red, green and blue light component or monochrome luminance (3 or 4 sub-sensors depending on the specific packaging) from an object illuminated with white light. The light is reflected by the object and pass through an aperture and a series of mirrors to create a focus length before it passes through a suitable lens that project it onto every single CCD sensor. Every sub-sensor have a color filter in front to filter out the Red, Green, Blue color information and an optional fourth line to capture monochromatic information only.

The light type currently used in scanner devices is the white light-emitting diode LED, that do not require long calibration and warming time.

Actually in the market there is no linear sensors available to capture objects larger than few inches. This imply that for scanning the Shroud we need to use a number of independent CCD subsystems, each having their own lenses and mirrors, linked together in a custom rigid sensor bar enough to cover the height of the Shroud (almost 48 inches).

This custom sensor bar will be calibrated with test patterns to discover and correct any kind of geometrical or radiometric errors that can occur, assuring the best quality for the scan operations.

\section{Project}

A project of a scanning line system must consider, as main requirements, the following ones:

a) acquisitions sensors shall be arranged along the width of the cloth; the movement of the sensors shall be in the direction of the cloth length;

b) variable spatial resolution: for the purposes of the body image treatment, aimed at highlighting evident and latent patterns, as well as to initiate studies on the genesis of the impression, the resolution shall be at least $600 \times 600 / 800 \times 800 \mathrm{dpi}$, or any mixing of them;

c) variable spectral resolution: we need to take the survey on different portions of the electromagnetic spectrum in order to specialize the recognition of information on the cloth; the spectral resolution shall be selectable during the scan operations;

d) radiometric resolution: the color depth for each RGB component shall be at least 8bits in order to obtain a sufficiently wide tonal range (this is a minimum requirement, but today sensors may detect at 12 or 16 bit per pixel).

The scanned image obtained with a system satisfying all the requirements above, could be classified as a scientific image for the following reasons:

a) the geometry aspects of the Shroud are respected, so we can do accurate metric evaluations;

b) the high spatial resolution of the image allows magnification, useful for depth processing and analysis;

c) colorimetric comparisons could be done in order to obtain classified pixel data.

A scientific image might provide researchers a new tool for analyzing the Shroud with an image quality comparable to a direct observation.

For the scanner device we may consider two different solutions, substantially constrained by the size of the environment available for the execution of scan tasks [6-8]:

a) translation of the Shroud, with scanner kept stationary;

b) translation of the scanner, with the Shroud held steady. 


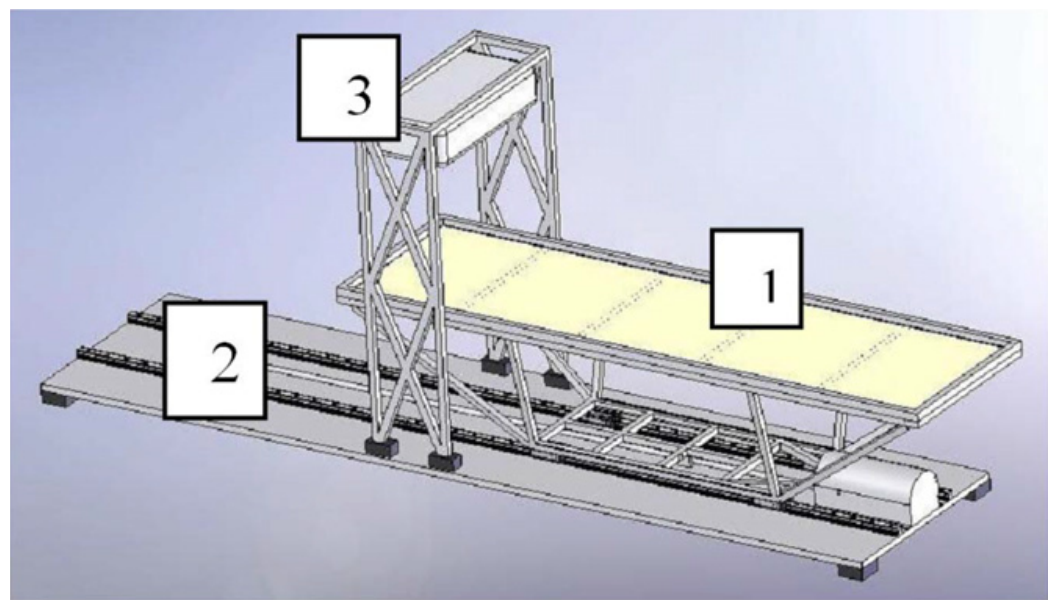

Figure 2. Overall scanning system with movement of the Shroud.

\subsection{System with the translation of the Holy Shroud}

This solution is favorable as regards the installation of the scanner, which must be placed in a fixed position, with obvious advantages regarding the stability of the device and the simplicity of the connections; this requires the development of a device for moving the Shroud that shows challenging aspects of space, stability and accuracy of movements.

For a correct execution of translation it is necessary to realize a mechanical system of the type shown in Fig. 2, which consists of the following main parts: (1) moving cart, (2) base for supporting and guiding the cart, (3) support structure of the scanner.

The scanning tasks will be performed in the following way:

- panel embodying the Shroud will be placed on the cart and bound to the latter in the same way it is usually bound to the bed ordinarily used for handling;

- carriage will be dragged along the rails of the base at constant speed;

- during the cart dragging, the scanner will acquire the image.

The following significant focus point should be highlighted:

- the complete translation of the Shroud under the scanner requires a working space of at least 9 meters

- the sliding guides of the carriage, and consequently the support base, must have at least length of 6.5 meters

- the supporting structure of the scanner must be high enough to place the unit at a distance of at least 1 meter from the Shroud

- the entire device needs to be placed on the floor by means of anti-vibration supports, in order to reduce any environmental vibration that could alter the scanning results.

The device design must take into account the following technical aspects:

- the cart must have a rigid and lightweight structure, materials and construction techniques will require proper investigation during the detailed phase of the project;

- the cart dragging must be uniform and free of vibrations; an optimal solutions with regard to the type of slide rails, the engine and the type of mechanical transmission is needed; 

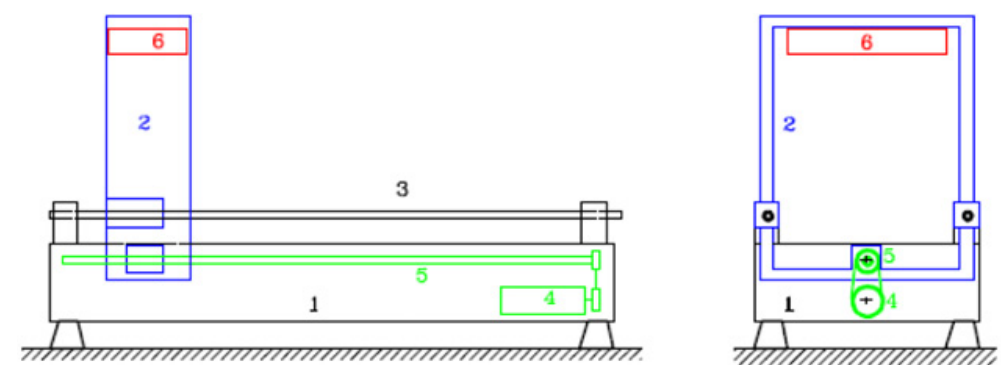

Figure 3. Global scanning movement of the trolley.

- the device as a whole must guarantee the complete absence of risks of damage to the Shroud; therefore it will avoid any contact with the cloth. All components for power generation or transformation (engine, mechanical transmission) must be equipped with safety devices in order to remove any electrical or mechanical risk;

- to enable the placement of the device in the room provided for the operations, an easily transportable and mountable structure is required.

The construction plan for the device building will be decomposed in the following way:

1) definition of characteristic requested for the scan device, especially with regard to the technical ones (speed, precision, sensing devices, ... ) and the positioning of the scanner;

2) project of the cart structure;

3) definition of the guiding elements for the cart;

4) definition of the type of engine to drive the cart;

5) definition of the mechanical transmission to drag the cart;

6) project of the support base of the device;

7) project of the fastening elements of the motor and the mechanical transmission;

8) project of the sensing system for relief of position and motion of the cart;

9) project of the support structure of the scanner and its links with the base of the device;

10) project the connection of the acquisition device to the data storage.

\subsection{System with translation of the scanner}

This solution ensures advantages with regard to the overall dimensions and the means to perform scanning operations, even if the environmental space is limited. In this way the Shroud stays stationary, and then the total length of the device is slightly greater than the length of the cloth. Figure 3 shows the diagram of a possible design solution.

The system consists of the following main parts:

- fixed base for placement of the Shroud and for housing components like the engine for driving the scanner cart;

- mobile gantry cart for scanner support;

- guidance system for carriage motion;

- motor;

- mechanical transmission;

- scanner. 
The scanning tasks are performed in the following way:

- the panel embodying the Shroud is placed on the base and bound to the latter in the same way it is usually bound to the bed currently used for the manual handling;

- carriage holding the scanner is dragged along the rails of the base;

- during the carriage drag the scanner will acquire the image.

The design of the device must take into account the following technical aspects:

- the base of the system will have a length of about 6 meters;

- the supporting structure of the scanner must be high enough to place the unit at a distance of at least 1 meter from the Shroud;

- the entire device will be placed on the floor by means of anti-vibration supports, in order to eliminate any environmental vibration that could alter the scan results;

- the cart must have a rigid structure and must be able to absorb any vibration: it will be necessary to properly study the materials and construction techniques;

- the cart dragging must be uniform and free of vibrations: an optimal solutions with regard to the type of slide rails, the engine and the type of mechanical transmission is needed;

- the device as a whole must guarantee the complete absence of risks to the Shroud; for this it will avoid any contact with the cloth. All components for power generation or transformation (engine, mechanical transmission) must be equipped with safety devices in order to remove any electrical or mechanical risk;

- to enable the placement of the device in the room provided for the operations, an easily transportable and mountable structure is desirable.

The criteria and design phases are similar to what has been shown for the solution with the translation of the Shroud.

\section{Conclusions}

Getting a single shot of the Shroud by means of a direct scan of the cloth, needs a dedicated device with extremely demanding performance which requires non-conventional design approach.

This proposal could be taken as a baseline for starting up a detailed project and plan for realizing such a goal.

\section{References}

[1] R.C. Gonzales, R.E. Woods, Digital image processing, Third edition (Prentice Hall, 2008)

[2] G.M. Zaccone, AA. VV., The two faces of the Shroud (Ed ODPF, 2001)

[3] N. Balossino, Sindone, immagini per la conoscenza (Effatà Editrice, Cantalupa - Torino, 2010)

[4] Chia Yen Chen, Image stitching comparison and New Techniques, CITR-TR-30 (1988)

[5] R. Szeliski, Image alignment and stitching: a tutorial, C.G. and Vision, Vol. 2. No.1 (2006)

[6] G. Belforte, Meccanica applicata alle macchine (Levrotto \& Bella, Torino, 2007)

[7] C. Ferraresi, T. Raparelli, Meccanica applicata (CLUT, Torino, 2007)

[8] E. Rivin, Mechanical design of robots (McGraw-Hill, New York, 1988) 